\section{Perturbed hematopoiesis in individuals with germline DNMT3A overgrowth Tatton-Brown-Rahman syndrome}

Ayala Tovy, ${ }^{1,2}$ Carina Rosas, ${ }^{1,2}$ Amos S. Gaikwad, ${ }^{3}$ Geraldo Medrano, ${ }^{3}$ Linda Zhang, ${ }^{1,2,4}$ Jaime M. Reyes, ${ }^{1,2,5}$ Yung-Hsin Huang, ${ }^{1}$ Tatsuhiko Arakawa, ${ }^{1,2}$ Kristen Kurtz ${ }^{3}$ Shannon E. Conneely, ${ }^{3}$ Anna G. Guzman, ${ }^{1,2}$ Rogelio Aguilar, ${ }^{3}$ Anne Gao, ${ }^{3}$ Chun-Wei Chen, ${ }^{1,2,4}$ Jean J. Kim ${ }^{1,2,6}$ Melissa T. Carter, ${ }^{7}$ Amaia Lasa-Aranzasti, ${ }^{8}$ Irene Valenzuela, ${ }^{8}$ Lionel Van Maldergem, ${ }^{9}$ Lorenzo Brunetti, ${ }^{310} \mathrm{M}$. John Hicks, ${ }^{11}$ Andrea N. Marcogliese, ${ }^{3}$ Margaret A. Goodell ${ }^{1,2,3,4,5,6 \#}$ and Rachel E. Rau ${ }^{1,3 \#}$

${ }^{1}$ Stem Cells and Regenerative Medicine Center, Baylor College of Medicine, Houston, TX, USA; ${ }^{2}$ Department of Molecular and Cellular Biology, Baylor College of Medicine, Houston, TX, USA; ${ }^{3}$ Department of Pediatrics, Baylor College of Medicine and Texas Children's Hospital, Houston, TX, USA; ${ }^{4}$ Graduate Program in Translational Biology and Molecular Medicine, Baylor College of Medicine, Houston, TX, USA; ${ }^{5}$ Department of Molecular and Human Genetics, Baylor College of Medicine, Houston, TX, USA; ${ }^{6}$ Department of Education, Innovation and Technology, Baylor College of Medicine, Houston, TX, USA; ${ }^{7}$ Department of Genetics, Children's Hospital of Eastern Ontario, Ottawa, Ontario, Canada; ${ }^{8}$ Department of Clinical and Molecular Genetics, Vall d' Hebron University Hospital and Medicine Genetics Group, Vall d`Hebron Research Institute, Barcelona, Spain; ${ }^{9}$ Centre de Génétique Humaine and Integrative and Cognitive Neuroscience Research Unit EA481, University of Franche-Comté, Besancon, France; ${ }^{10}$ Department of Medicine and Surgery, University of Perugia, Perugia, Italy and ${ }^{11}$ Department of Pathology Texas Children's Hospital and Department of Pathology and Immunology, Baylor College of Medicine, Houston, TX, USA

"MAG and RER contributed equally as co-senior authors.

\section{ABSTRACT}

T atton-Brown-Rahman syndrome (TBRS) is an overgrowth disorder caused by germline heterozygous mutations in the DNA methyltransferase DNMT3A. DNMT3A is a critical regulator of hematopoietic stem cell (HSC) differentiation and somatic DNMT3A mutations are frequent in hematologic malignancies and clonal hematopoiesis. Yet, the impact of constitutive DNMT3A mutation on hematopoiesis in TBRS is undefined. In order to establish how constitutive mutation of DNMT3A impacts blood development in TBRS we gathered clinical data and analyzed blood parameters in 18 individuals with TBRS. We also determined the distribution of major peripheral blood cell lineages by flow cytometric analyses. Our analyses revealed non-anemic macrocytosis, a relative decrease in lymphocytes and increase in neutrophils in TBRS individuals compared to unaffected controls. We were able to recapitulate these hematologic phenotypes in multiple murine models of TBRS and identified rare hematological and non-hematological malignancies associated with constitutive Dnmt3a mutation. We further show that loss of DNMT3A in TBRS is associated with an altered DNA methylation landscape in hematopoietic cells affecting regions critical to stem cell function and tumorigenesis. Overall, our data identify key hematopoietic effects driven by DNMT3A mutation with clinical implications for individuals with TBRS and DNMT3A-associated clonal hematopoiesis or malignancies.

\section{Introduction}

Tatton-Brown-Rahman syndrome (TBRS; OMIM: 615879) is a germline dominant disorder due to constitutive heterozygous mutations in the de novo DNA methyltransferase DNMT3A. Individuals with TBRS are typically tall, obese, macrocephalic and have varying degrees of intellectual disability., Thus far, approximately 60 TBRS individuals have been described in the literature. However, the number of diagnosed indi-
Haematologica 2022

Volume 107(4):887-898

\section{Correspondence:}

RACHEL E. RAU

rachel.rau@bcm.edu

MARGARETA. GOODELL

goodell@bcm.edu

Received: April 14, 2021.

Accepted: May 21, 2021.

Pre-published: June 3, 2021.

https://doi.org/10.3324/haematol.2021.278990

(C)2022 Ferrata Storti Foundation

Material published in Haematologica is covered by copyright. All rights are reserved to the Ferrata Storti Foundation. Use of published material is allowed under the following terms and conditions:

https://creativecommons.org/licenses/by-nc/4.0/legalcode. Copies of published material are allowed for personal or internal use. Sharing published material for non-commercial purposes is subject to the following conditions:

https://creativecommons.org/licenses/by-nc/4.0/legalcode, sect. 3. Reproducing and sharing published material for commercial purposes is not allowed without permission in writing from the publisher. 
viduals is steadily increasing with growing awareness of DNMT3A mutations as a cause of overgrowth and developmental disorders. As the full extent of TBRS clinical features is not yet defined, ongoing efforts to characterize the effects of constitutive DNMT3A loss will be critical to facilitate identification of affected individuals and improve their care and quality of life.

DNMT3A is perhaps best known as a regulator of blood development governing the balance between hematopoietic stem cell (HSC) self-renewal and differentiation. ${ }^{3}$ Loss-offunction DNMT3A mutations are highly prevalent across a spectrum of adult hematologic malignancies, further confirming its indispensable role in hematopoiesis. ${ }^{4-6}$ In addition, DNMT3A is the most commonly mutated gene in clonal hematopoiesis $(\mathrm{CH}),{ }^{7,8}$ a phenomenon of aging associated with mutant hematopoietic stem and progenitor cell (HSPC) expansion. ${ }^{9}$ Individuals with $\mathrm{CH}$ have a significantly increased risk for hematologic malignancy development. This increased risk is likely due to a competitive growth advantage that leads to an accumulation of DNMT3Amutant HSPC in the bone marrow over time, ${ }^{3}$ with a proportional increase in the likelihood of acquiring collaborating leukemogenic mutations. Consistent with this timedependent model of clonal expansion and malignant transformation is the fact that, while common in adult hematologic malignancies, DNMT3A mutations are exceedingly rare in pediatric leukemias. ${ }^{10-13}$

The mutation spectrum reported for TBRS individuals covers all the functional domains of DNMT3A and overlaps with $\mathrm{CH}$ and hematologic malignancies. ${ }^{1}$ However, in contrast to the somatic mutations in $\mathrm{CH}$ and cancer, in TBRS individuals, the DNMT3A mutation is constitutive, arising before embryogenesis. This timing raises the concern that the negative hematopoietic sequelae associated with somatic DNMT3A mutations in older individuals could have an early onset in TBRS. Reports of recurrent infections, bleeding tendencies, and leukemias in individuals with TBRS suggest that there may be a correlation between constitutive DNMT3A loss and abnormal hematopoiesis. ${ }^{1,14,15}$ Despite these concerns and anecdotal observations, how constitutive loss of DNMT3A impacts blood formation and leukemia predisposition in TBRS remains unknown.

In order to define basal hematopoiesis in TBRS, we extensively characterized the hematological parameters and peripheral blood cellular composition of TBRS individuals with a spectrum of DNMT3A mutations and deletions. We found that germline DNMT3A mutations impact multilineage blood development, resulting in clinically relevant phenotypes. We further identified DNA methylation changes as one mechanism through which constitutive loss of DNMT3A alters blood development in TBRS. In order to confirm the observed hematopoietic effects, we extensively investigated hematopoiesis in multiple mouse models of TBRS. These murine models recapitulated the major hematologic phenotypes identified in humans. Longitudinal studies of the TBRS mouse models demonstrated that while the risk of hematologic malignancies is increased in TBRS, disease penetrance overall is low.

\section{Methods}

\section{Human samples}

This study was approved by the Institutional Review Board of Baylor College of Medicine. Written informed consent and assent where appropriate were obtained from each participant and/or his or her guardian in accordance with the declaration of Helsinki prior to any study procedures. Peripheral blood samples from individuals with germline DNMT3A mutations or deletions and unaffected controls were collected in EDTA-coated tubes. Available medical records for enrolled patients were reviewed for growth parameters, laboratory studies and hematologic diagnoses.

\section{Mouse models}

All mice were housed in AAALAC-accredited, specificpathogen-free animal care facilities at Baylor College of Medicine, and all procedures were approved by the Institutional Animal Care and Use Committee. C57BL/6 mice of both sexes were used unless stated otherwise, and experimental mice were separated by sex and housed with four mice per cage. All mice were immune-competent and healthy prior to the experiments described. Mice were bred and maintained at regular housing temperatures $\left(23^{\circ} \mathrm{C}\right)$ and 12 -hour $(\mathrm{h})$ light/12h dark cycle. In order to generate germline Dnmt3a heterozygous haploinsufficient mice we crossed $D n m t 3^{+/ l l}$ mice with Ellacre transgenic mice obtained from The Jackson Laboratories (Bar Harbor, ME). The DNMT3A p.293 deletion and DNMT3A p.W577R mutation were generated in our laboratory utilizing CRISPR strategies with a single guide RNA and single strand DNA template injected into mouse blastocysts. ${ }^{16}$ Guide sequence and DNA template are described in the Online Supplementary Table S3. For all assays mouse data was obtained at 12 or 15 months of age.

\section{Lymphoblastoid cells generation}

Peripheral blood mononuclear cells were isolated from whole blood by density gradient centrifugation using Lymphoprep density gradient medium (STEMCELL Technologies, Vancouver, Canada) and then incubated with concentrated Epstein-Barr virus in a total of $200 \mu \mathrm{L}$ of RPMI medium for 30 minutes. The cells were then plated in a flat-bottomed 96-well plate with $1 \mu \mathrm{g} / \mathrm{mL}$ cyclosporin A (Sandoz Pharmaceuticals, Washington, DC). Cells were fed bi-weekly until lymphoblastoid cell lines (LCL) were established.

\section{Whole genome bisulfite sequencing}

DNA was extracted from LCL using the DNeasy kit (Qiagen, Hilden, Germany). DNA was fragmented prior to bisulfite conversation using the NEBNext fragmentase kit, following the manufacturer's protocol (New England BioLabs, Inc., Ipswich, MA). We used $200 \mathrm{ng}$ of DNA to prepare whole genome bisulfite sequencing (WGBS) libraries with the Swift-whole genome bisulfite kit according to the manufacturer's instruction (Swift Biosciences, Ann Arbor, MI). Libraries were sequenced and processed as previously described. ${ }^{17}$ The Wildcard ALignment tool (WALT) pipeline was used for duplicate removal and hypomethylated regions (HMR) calling. ${ }^{18}$ A minimum of five reads per $\mathrm{CpG}$ was used to calculate methylation values. Coverage per base for mutant or control cells was $>7 x$. For identification of differentially methylated regions, we used the WALT pipeline default settings.

\section{Statistical analyses}

Statistical analyses of the clinical data were performed with the GraphPad Prism 8 software (GraphPad Software, San Diego, CA). $P$-values were interpreted as statistically significant if less than 0.05 , unless otherwise stated. Throughout the manuscript data are expressed as the mean $+/$ - standard error of the mean, unless otherwise stated. The statistical significance of the 


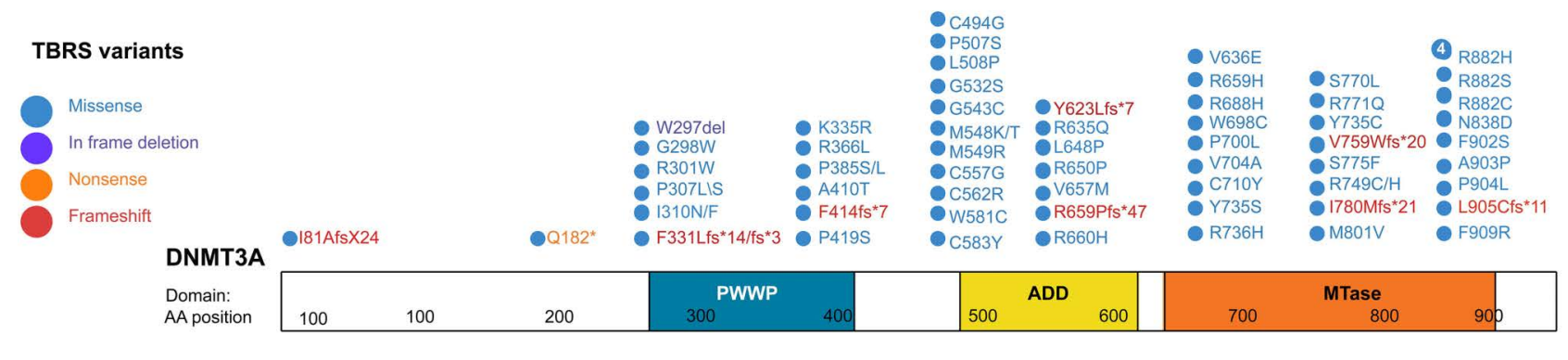

Figure 1. DNMT3A mutations and deletions identified in patients with Tatton-Brown-Rahman syndrome. Map of DNMT3A variants identified in individuals with Tatton-Brown-Rahman syndrome (TBRS) based on clinical sequencing data from our patient cohort, published data, and information obtained from the TBRS community. AA: amino acid; PWWP: Pro-Trp-Trp-Pro motif domain; ADD: ATRX-DNMT3L-DNMT3L domain; Mtase: methyltransferase domain.

differences between two groups was calculated using unpaired Student's $t$-test (two-sided, without assuming equal standard deviations), Fisher's exact test or the nonparametric MannWhitney test where appropriate. Statistical details are also described in the figure legends, including the number of replicates, animals or human samples per group (denoted by "n").

\section{Results}

\section{Characteristics of germline DNMT3A mutations in Tatton-Brown-Rahman syndrome}

In order to identify the current spectrum of TBRS variants, we gathered mutational information on 48 individuals with TBRS utilizing the TBRS organization database. These individuals harbored mutations (missense, frameshift, deletions) in the PWWP, ADD and methyltransferase domains, including $\sim 30$ not previously reported in TBRS (Figure 1, Table 1; Online Supplementary Table S1). We also identified two TBRS individuals with deletion of the entire DNMT3A gene. These data show that, as previously suggested, DNMT3A mutations in TBRS can occur across all the DNMT3A functional domains and are shared between TBRS, CH and leukemia.

\section{White blood cell differential of Tatton-Brown-Rahman syndrome individuals is characterized by increased neutrophils and decreased lymphocytes}

In order to assess the composition of the blood of individuals with TBRS, we enrolled 18 individuals with TBRS, seven unaffected siblings, and four unrelated unaffected individuals (Table 1; Online Supplementary Table S2). The clinical and genetic information for the included TBRS individuals is shown in Table 1. One individual in the TBRS cohort had severe iron deficiency anemia and one had hypogammaglobulinemia requiring intravenous immunoglobulin (IVIG) replacement. No other blood or immune defects were reported in either cohort.

We performed complete blood cell counts (CBC) with white blood cell (WBC) differential on the enrolled individuals, excluding from the analysis the TBRS patient with severe iron deficiency anemia. An additional four TBRS individuals and one control did not have a CBC performed, thus 13 TBRS individuals and nine controls were included in our analysis. The age of TBRS individuals (median 15.2 years, range, 3.5-35.8 years) with CBC was not significantly different than controls (median 10.0 years, range, 3.5-40 years).

We found that while the total WBC count was not dif- ferent between the groups, in TBRS individuals the percentage of neutrophils was significantly increased, and percentage of monocytes and lymphocytes significantly decreased relative to controls (Figure 2A to D). Also, while hemoglobin and red cell counts (RBC) were not significantly different (Figure 2E and F) compared to unaffected individuals, those with TBRS had a significantly increased mean corpuscular volumes (MCV) and mean corpuscular hemoglobin $(\mathrm{MCH})$ levels, with no difference in mean corpuscular hemoglobin concentration (MCHC) (Figure 2G; Online Supplementary Figure 1; Figure $2 \mathrm{H}$, respectively). As MCV varies by age and sex, we plotted the MCV of our enrolled TBRS individuals by age/sex, including past CBC from medical records where available. We found that overall, the vast majority of TBRS individuals had MCV above the $50^{\text {th }}$ percentile at all ages examined including ten of 14 with an $M C V \geq 90^{\text {th }}$ percentile and eight of $14>97^{\text {th }}$ percentile for age/sex at one or more timepoints (Figure 2I and J). All other CBC parameters were similar between TBRS and unaffected individuals (Online Supplementary Figure 1). These finding indicate that heterozygous germline DNMT3A lesions impact multilineage hematopoiesis in the absence of overt clinical blood disorders.

\section{Analytical characterization of blood populations in Tatton-Brown-Rahman syndrome individuals}

We next conducted immunophenotypic analyses of the peripheral blood from 15 TBRS individuals and ten controls. The age of TBRS versus control individuals with immunophenotypic data was not significantly different (median 13.5 years and 10 years, respectively). Our findings confirmed neutrophil expansion in the blood of TBRS individuals (Figure 3A). Given reports of recurrent infections and hypogammaglobulinemia in individuals with TBRS as well as mouse model data suggesting a role for DNMT3A in T-cell development, ${ }^{19}$ we conducted a detailed immunophenotypic analysis of $\mathrm{T}$ - and $\mathrm{B}$-cell subsets. We found a relative reduction in $\mathrm{CD} 19^{+}, \mathrm{CD} 20^{+}$, and $\mathrm{CD} 22^{+} \mathrm{B}$ cells in TBRS individuals compared to unaffected controls (Figure 3B; Online Supplementary Figure $S 2 A$ and $B$ ). We also identified a trend towards reduced total $\mathrm{CD}^{+} \mathrm{T}$ cells that did not reach statistical significance but found significant differences in the relative populations of $\mathrm{CD}^{+}$and $\mathrm{CD}^{+}$expressing $\mathrm{T}$ cell subsets with a higher CD4 to CD8 ratio in TBRS compared to unaffected individuals (Figure 3C; Online Supplementary Figure 2C). These results confirm a relative increase in neutrophils in individuals with TBRS and identify significant changes in $\mathrm{B}$ - and $\mathrm{T}$-cell populations. 
Table 1. Table of Tatton-Brown-Rahman syndrome individuals' clinical and mutation information.

\begin{tabular}{|c|c|c|c|c|c|c|c|c|c|c|c|}
\hline D & Sex & $\begin{array}{l}\text { Age } \\
\text { (vears) }\end{array}$ & $\begin{array}{l}\text { Height } \\
\text { (cm) }\end{array}$ & $\begin{array}{l}\text { Height } \\
\text { percentile }\end{array}$ & $\begin{array}{l}\text { Weight } \\
\text { (kg) }\end{array}$ & $\begin{array}{l}\text { Weight } \\
\text { percentile }\end{array}$ & $\begin{array}{l}\text { Mutation } \\
\text { type }\end{array}$ & $\begin{array}{l}\text { DNMT3A coding } \\
\text { sequence variant }\end{array}$ & $\begin{array}{l}\text { DNMT3A } \\
\text { variant protein }\end{array}$ & Flow Y/N & CBC \\
\hline RR01 & M & 3.8 & 110.4 & 96 & 19.9 & 99 & Missense & c. $901 \mathrm{C}>\mathrm{T}$ & p.R301W & Y & Y \\
\hline RR04 & M & 21.8 & 183 & 83 & 89 & 55 & Frameshift deletion & c.297del & p.M99fs & $\mathrm{N}$ & $\mathrm{N}$ \\
\hline RR07 & M & 2.6 & 104.8 & $>99$ & 26.2 & $>99$ & Gene deletion & 2p23.3 del & & Ex & Ex \\
\hline RR16 & M & 17.6 & 183 & 84 & 79.5 & 84 & Missense & c. $1748 \mathrm{G}>\mathrm{A}$ & p.C583Y & $\mathrm{Y}$ & Y \\
\hline RR17 & M & 34.5 & 196 & $>99$ & 79.4 & 35 & Frameshift duplication & c.1238dupG & p.F414fs*7 & Y & Y \\
\hline RR18 & M & 20.8 & 196 & $>99$ & 74.4 & 23 & Missense & c. $2309 \mathrm{C}>\mathrm{T}$ & p.S770L & Y & Y \\
\hline RR19 & $\mathrm{F}$ & 35.8 & 194 & $>99$ & 77.5 & 30 & Missense & c. $2207 \mathrm{G}>\mathrm{A}$ & p.R736H & $\mathrm{Y}$ & Y \\
\hline RR20 & $\mathrm{F}$ & $16^{*}$ & 172 & 93 & 68.5 & 88 & Missense & c. $2063 \mathrm{G}>\mathrm{A}$ & p.A688H & Y & Y \\
\hline RR22 & M & 3.5 & 99 & 47 & 17.7 & 87 & Missense & c. $1978 \mathrm{~T}>\mathrm{C}$ & p.Y660H & $\mathrm{Y}$ & Y \\
\hline RR24 & M & 5.7 & 130 & $>99$ & 33.1 & $>99$ & Gene deletion & 2p23.3 del & & Y & Y \\
\hline RR25 & M & 13.5 & 168 & 78 & 83.9 & 99 & Missense & c. $2645 \mathrm{G}>\mathrm{A}$ & p.R882H & Y & Y \\
\hline RR26 & $\mathrm{F}$ & 16.0 & 180.3 & $>99$ & 115.5 & 99 & Missense & c. $929 \mathrm{~T}>\mathrm{A}$ & p.I310N & Y & Y \\
\hline RR28 & $\mathrm{F}$ & $18^{*}$ & 168 & 77 & 64 & 75 & Missense & c. $2114 \mathrm{~T}>\mathrm{C}$ & p.V704A & $\mathrm{N}$ & $\mathrm{N}$ \\
\hline RR34 & M & 5.9 & $\mathrm{n} / \mathrm{k}$ & $\mathrm{n} / \mathrm{k}$ & $\mathrm{n} / \mathrm{k}$ & $\mathrm{n} / \mathrm{k}$ & Missense & c.2129G & p.C710Y & Y & Y \\
\hline RR38 & $\mathrm{F}$ & 7.0 & 137 & $>99$ & 30 & 91 & Missense & c. $919 \mathrm{C}>\mathrm{T}$ & p.P307S & Y & Y \\
\hline RR40 & M & 15.2 & 193 & $>99$ & 74.5 & 89 & $\begin{array}{c}\text { Frameshift deletion/ } \\
\text { insertion }\end{array}$ & .2432_2434delinsC & & Y & $\mathrm{Y}$ \\
\hline RR43 & $\mathrm{F}$ & 2.0 & 93 & 99 & 15 & 97 & Missense & c. $1627 \mathrm{G}>\mathrm{T}$ & p.G543C & $Y$ & $\mathrm{~N}$ \\
\hline RR45 & M & 3.7 & 107 & 92 & 21 & 98 & Missense & c. $2645 \mathrm{G}>\mathrm{A}$ & p.R882H & Y & $\mathrm{N}$ \\
\hline
\end{tabular}

Blood and medical records we collected from 18 Tatton-Brown-Rahman syndrome (TBRS) individuals. Presented is mutation data and the type of analyses that were done on each sample. * approximate; n/k: not known; Flow: flow cytometric analysis of peripheral blood; CBC: complete blood cell count with white blood cell differential;Y: yes; N: no; Ex: excluded due to severe iron deficiency anemia.

\section{Myeloid cells expand in the peripheral blood of mice with germline Dnmt3a mutations}

In order to validate the findings of our human cohort, we examined hematopoiesis in three mouse models with constitutive Dnmt3a lesions. We first evaluated mice with a heterozygous germline deletion affecting amino acid 293 (HET293) (mouse equivalent of human amino acid 297) in the PWWP domain of DNMT3A, a lesion previously reported in TBRS. ${ }^{1,2}$ Consistent with our human data, CBC revealed significantly increased percentage of neutrophils and decreased lymphocytes compared to wildtype littermate controls (WT) without a significant difference in the overall WBC count (Figure 4A to C; Online Supplementary Figure S3A).

In order to evaluate for potential phenotype-modifying effects of differing Dnmt3a mutations on hematopoiesis, we also analyzed the peripheral blood of mice with heterozygous germline mutations affecting the DNMT3A ADD domain amino acid W577R (HET577) (mouse equivalent of human W581R), a mutation which has been reported in $\mathrm{CH}^{20}$ and mice with heterozygous deletion of Dnmt3a (HET), recapitulating a complete loss of function or deletion of one DNMT3A copy. At 1 year of age, we compared the peripheral blood of HET577 and HET mice to their WT littermates and found that the percentage of neutrophils was significantly increased whereas the percentage of lymphocytes was significantly decreased (Online Supplementary Figure $3 B$ and C). HET577 mice also had significantly decreased total WBC and decreased platelets, unlike the HET293 and HET mice and the TBRS cohort.

Flow cytometric immunophenotypic analysis of peripheral blood leukocytes at 12 and 15 months demonstrated significant myeloid expansion and B-cell reduction in the HET293 mice and HET577 (Figure 4D to F; Online
Supplementary Figure S4A and B, respectively) consistent with findings in our TBRS cohort. Like TBRS individuals, the percentage of Ly6G+ neutrophils in HET293 mice was significantly increased relative to littermate controls with no significant difference in the percentage of Ly6C+ monocytes (Figure 4G). Together, these results demonstrate that multiple constitutive Dnmt3a mutant mouse models recapitulate the hematopoietic phenotypes observed in TBRS.

Previous publications have shown that the key inflammatory cytokine, interleukin 6 (IL6), is abnormally elevated in DNMT3A-mediated $\mathrm{CH}^{21}$ We therefore postulated that the neutrophil expansion identified in TBRS and our murine models may be in response to inflammation driven by mutation or deletion of Dnmt3a. In order to explore this possibility, we measured IL6 levels in the serum of HET293 mice and WT littermates at 12 months and in the TBRS individuals. Although in the young TBRS individuals IL6 was not altered, in HET293 mice we measured significantly elevated levels of IL6 (Online Supplementary Figure 4C and $D$ ). Our findings suggest that germline Dnmt3a mutations are consistently associated with neutrophil expansion, potentially in response to heightened inflammation.

\section{Stem and myeloid cells expand in bone marrow of aged germline Dnmt3a-mutant mice}

Knockout (KO) of Dnmt3a in hematopoietic stem cells has been widely investigated in bone marrow transplantation experiments, in which transplant of Dnmt3a-KO HSC leads to expansion of the stem cell pool and increased repopulation capability. ${ }^{22}$ The impact of DNMT3A mutation or loss on hematopoietic stem and progenitor cell populations in an unperturbed model such as TBRS has not been fully investigated. In order to address this we next analyzed the composition of hematopoietic cells in 
A

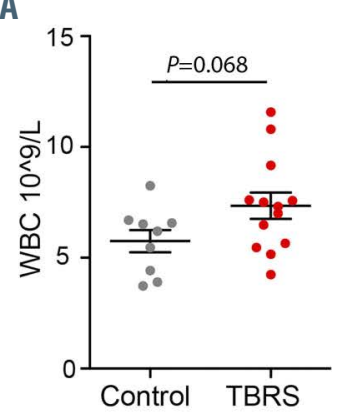

B

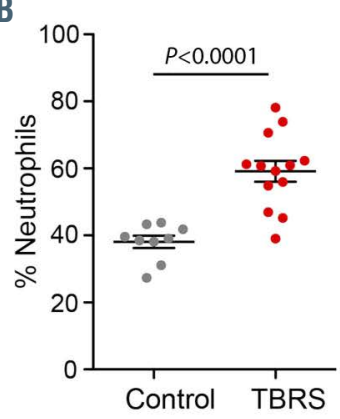

C

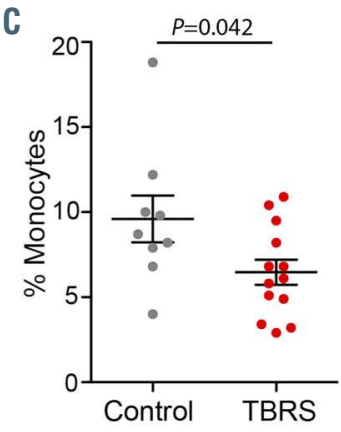

D

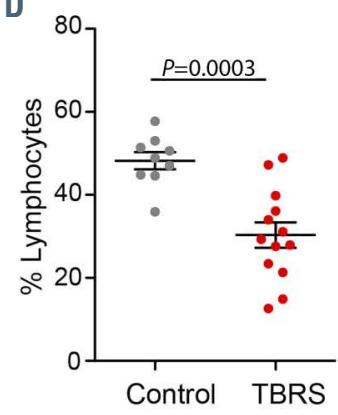

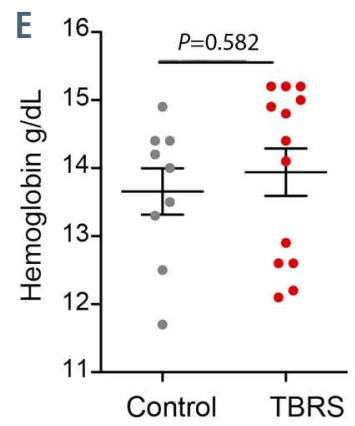

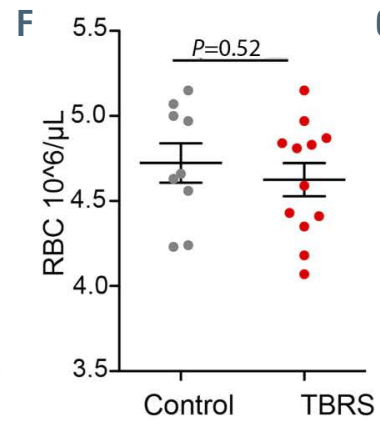

G

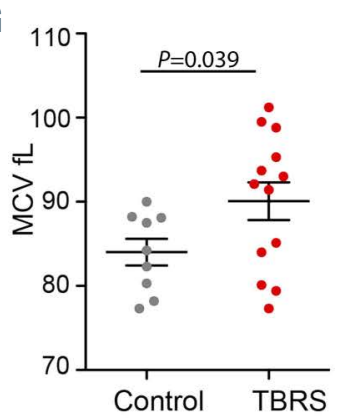

H

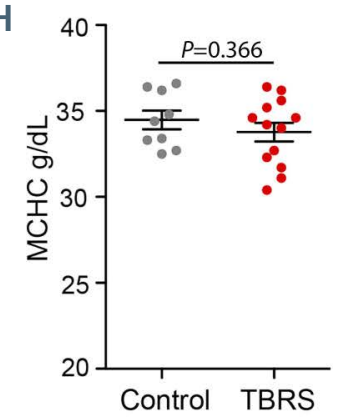

I

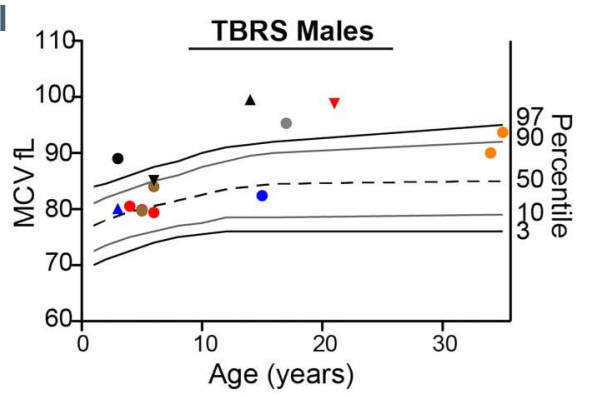

- RR1 - RR24

- RR16 A RR25

- RR17 vRR34

VRR18 • RR40

$\triangle \mathrm{RR} 22$ - RR45

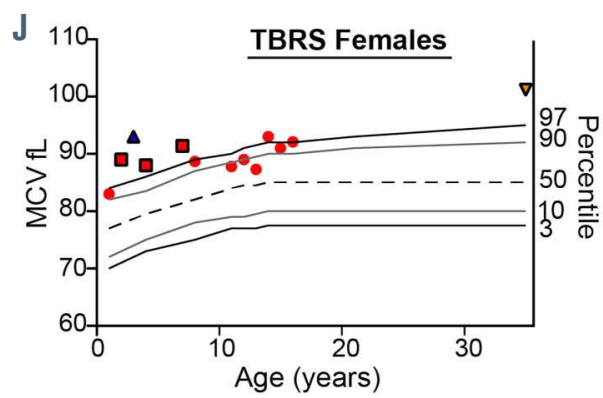

$\boldsymbol{\nabla}$ RR19

$\triangle \mathrm{RR} 20$

- RR26

- RR38

Figure 2. Blood of Tatton-Brown-Rahman syndrome individuals is characterized by relative increase in neutrophils, decrease in lymphocytes and non-anemic macrocytosis. Complete blood cell counts were performed on peripheral blood from Tatton-Brown-Rahman syndrome (TBRS) individuals ( $\mathrm{n}=13$ ) and unaffected controls ( $n=9$ ) including (A) total white blood cell (WBC) count and WBC differential with percent of (B) neutrophils, (C) monocytes, and (D) lymphocytes. Red blood cell (RBC) indices were also compared including (E) hemoglobin, (F) total RBC number, (G) mean corpuscular volume (MCV), and (H) mean corpuscular hemoglobin concentration (MCHC). One TBRS individual had no available RBC count. MCV plotted by age for (I) TBRS males and (J) TBRS females including data from study CBC and from $\mathrm{CBC}$ data extracted from medical records for some individuals over multiple timepoints.

the bone marrow of HET293 mice compared to WT littermate controls. Like the blood, the bone marrow of HET293 mice showed relative myeloid expansion and reduced frequency of B-cells (Figure $4 \mathrm{H}$ to J). Comparison of the stem/progenitor cell compartment (gating scheme in the Online Supplementary Figure $S 4 E$ and F) in 15 -monthold mice without any overt hematologic malignancies, showed a moderate but significant expansion of hematopoietic stem cells and multipotent progenitor cells (Figure $4 \mathrm{~K}$ and $\mathrm{L}$ ). The relative frequency of other stem/progenitor populations, including common myeloid progenitors, common lymphoid progenitors, granulocytes/monocyte progenitors, megakaryocyte/erythroid progenitors did not differ significantly (Online Supplementary Figure $4 G$ and $H$ ).
These finding support that the germline mutation of Dnmt3a, like somatic loss of Dnmt3a in the hematopoietic compartment, results in stem cell expansion with aging and leads to myeloid expansion in the bone marrow. ${ }^{3}$

\section{Germline Dnmt3a-mutant mice have defects in lymphocytes production}

Evaluation of T- and B-cell subsets in the blood of TBRS individuals revealed significantly reduced $\mathrm{CD} 19^{+} \mathrm{B}$ cells and an altered ratio of CD4/CD8 $\mathrm{T}$ cells in those with TBRS. We therefore performed flow cytometric analysis of B- and T-cell subsets in the peripheral blood of Dnmt3a-mutant mice. We found that unlike TBRS individuals, in HET293 and HET577 mice the CD4/CD8 T-cell ratio was significantly lower than WT controls (Online 
A

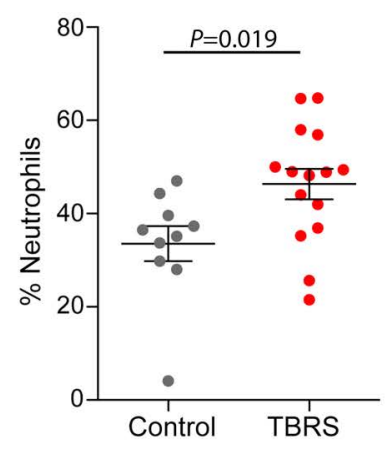

C

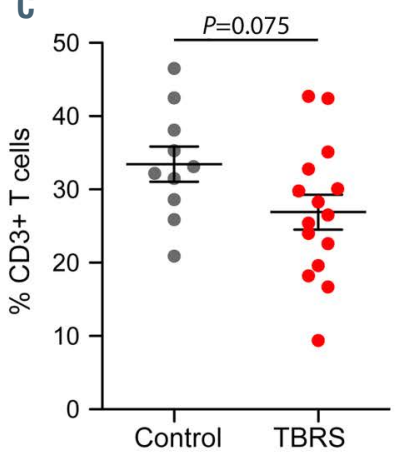

B

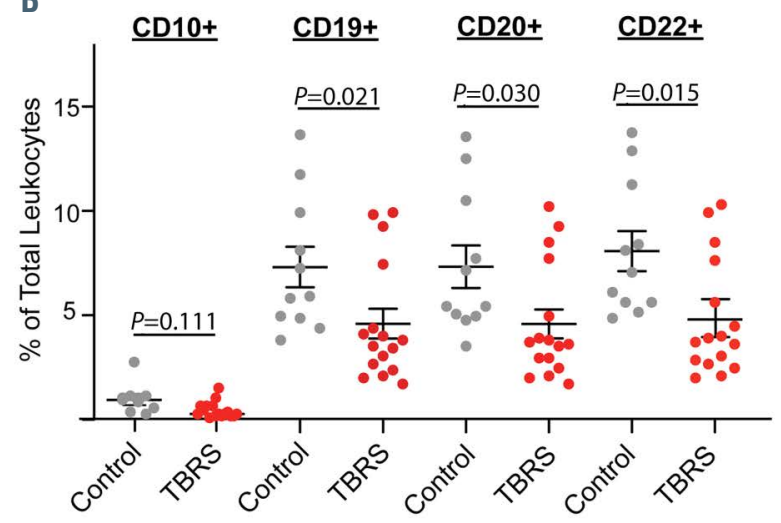

.

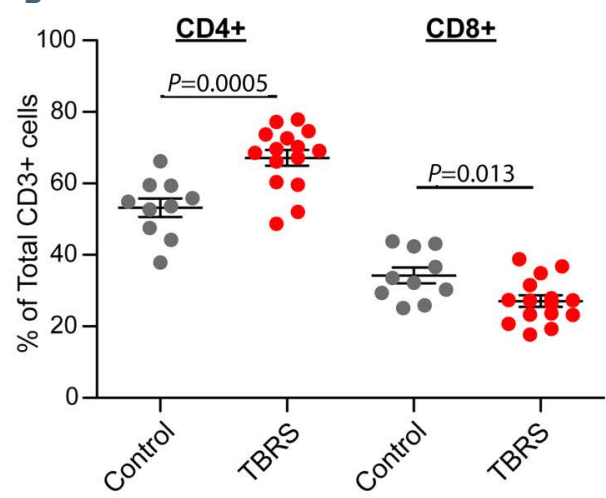

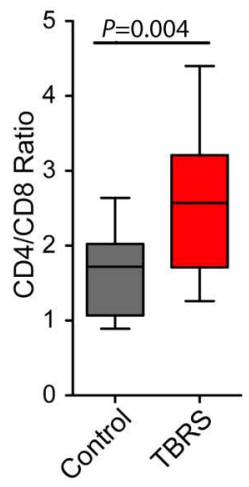

Figure 3. Immunophenotypic analysis of Tatton-Brown-Rahman syndrome individuals identifies neutrophil expansion and deficiencies in specific T- and B- cell subsets. Flow cytometry analysis was performed on peripheral blood from Tatton-Brown-Rahman syndrome (TBRS) individuals $(n=15)$ and unaffected controls ( $n=10)$. Percent of leukocytes categorized by immunophenotype as (A) neutrophils, (B) B cells expressing CD10, CD19, CD20 and/or CD22, (C) total CD3 ${ }^{+}$T cells and the percentage of $\mathrm{CD}^{+} \mathrm{T}$ cells that are $\mathrm{CD}^{+}$and $\mathrm{CD}^{+}$. (D) Quantification of the $\mathrm{CD} 4 / \mathrm{CD} 8$ ratio of controls versus TBRS individuals.

Supplementary Figure S5A). Quantification of B-cell subsets by flow cytometry demonstrated that although HET293 mice had a relative decrease in total $\mathrm{B} 220^{+} \mathrm{B}$ cells, the proportion of different B-cell subsets was not altered when compared to WT mice (Online Supplementary Figure S5B). Only mature B lymphocytes can effectively contribute to the immune response, and maturation of B-cells that occurs in the spleen. Therefore, in order to determine if the B-cell reduction reflects defects in splenic B-cell maturation we analyzed the frequency of transitional immature $B$ cells in the spleen by flow cytometry (T1 and T2 populations, respectively). ${ }^{23} \mathrm{~T} 1 \mathrm{~B}$ cells are bone marrow derived immature B cells that migrate to the spleen where they mature into T2 B cells which are the progenitors for mature B cells. ${ }^{24}$ We identified a significant decrease in the frequency of T1 B cells but not of T2 B cells in the spleen of HET293 mice compared to WT controls (Figure $5 \mathrm{~A}$ and $\mathrm{B})$. These data suggest that DNMT3A loss leads to a reduction in B-cell frequency via decreased B-cell progenitors in the bone marrow. While reduced in numbers, these progenitors are capable of normal maturation.

\section{Germline Dnmt3a-mutant mice have defects in erythropoiesis}

Evaluation of RBC indices in our TBRS cohort revealed increased average MCV and MCH relative to unaffected individuals. This finding was recapitulated in the HET293, HET577, and HET murine models (Figure 5D and E; Online Supplementary Figure S3), although unlike the TBRS individuals, HET293 mice additionally had significantly decreased RBC counts compared to WT controls (Figure $5 \mathrm{C}$ to $\mathrm{E})$. In order to further characterize erythropoiesis in TBRS, we performed flow cytometric examination of erythroid development in the bone marrow of HET293 mice. ${ }^{25}$ Staining of bone marrow cells with CD71 and TER119 is used to identify developmental stages of erythropoiesis, labeled ProE (proerythroblasts), Erythroblasts A-C (EryA, EryB and EryC), which correspond to sequential steps in erythroid development. ${ }^{26}$ Although the total percentage of TER119 positive cells was not significantly different in HET293 mice compared to WT littermates, HET293 mice had significantly fewer large, immature erythroblasts (EryA) and more small mature erythroblasts (EryC) (Figure 5F and G). Thus, loss of DNMT3A in our murine model leads to a subtle, but significant effect on early erythroid development and differentiation.

\section{Dnmt3a-mutant mice develop hematologic malignancies at higher rates than controls}

Given the frequency of somatic DNMT3A mutations in adult hematologic malignancies, as well as case reports documenting hematologic malignancies in individuals with TBRS, 1,14 we looked for malignancies in cohorts of HET293 mice at the age of 15 months. At this age, eight of $36(22 \%)$ HET293 mice had malignancies compared to one of $35(3 \%)$ WT littermate controls $(P=0.028)$. Of the eight malignancies in the HET293 mice, seven were hematologic including both myeloid and lymphoid diseases (Online Supplementary Table S7; Online Supplementary Figure 6 A and $B)$ consistent with results from mice with heterozygous 

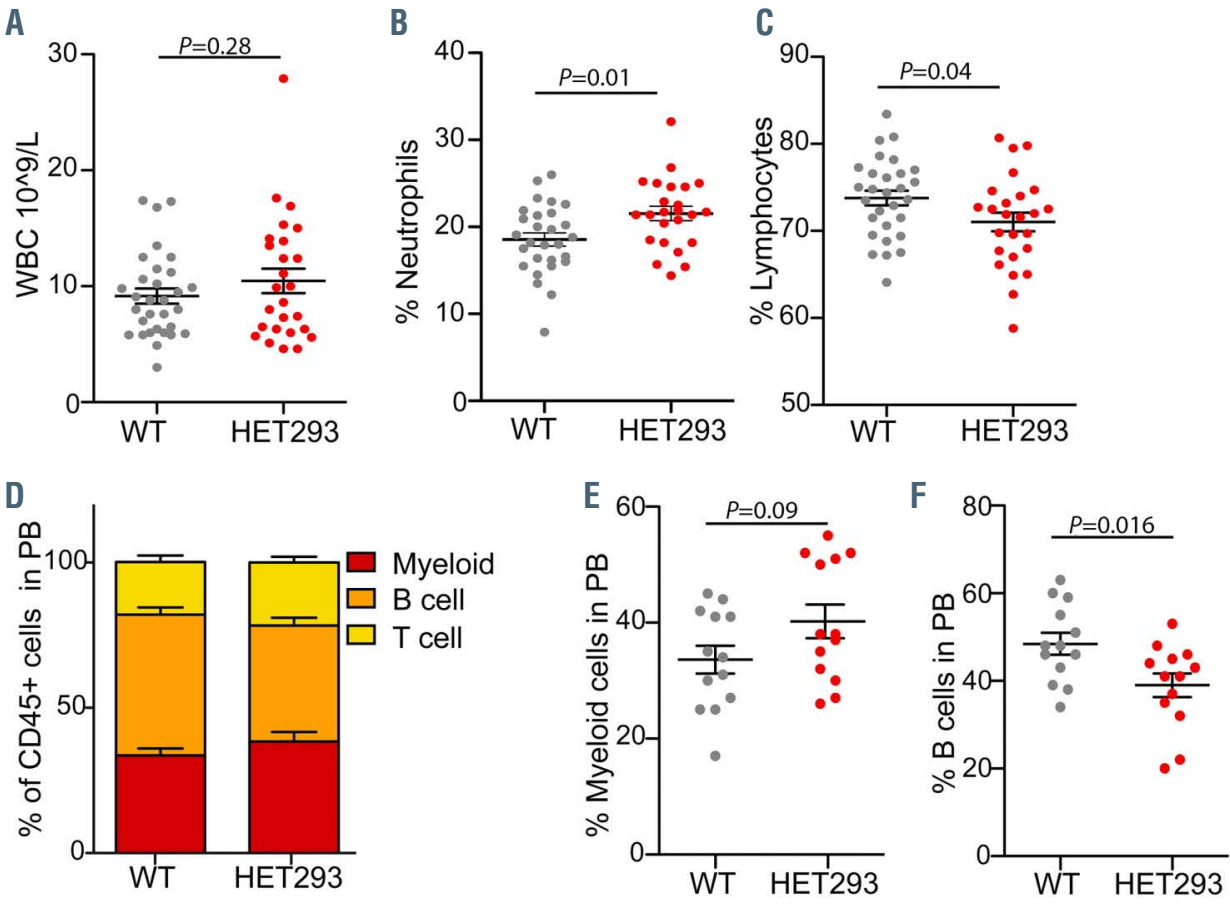

G

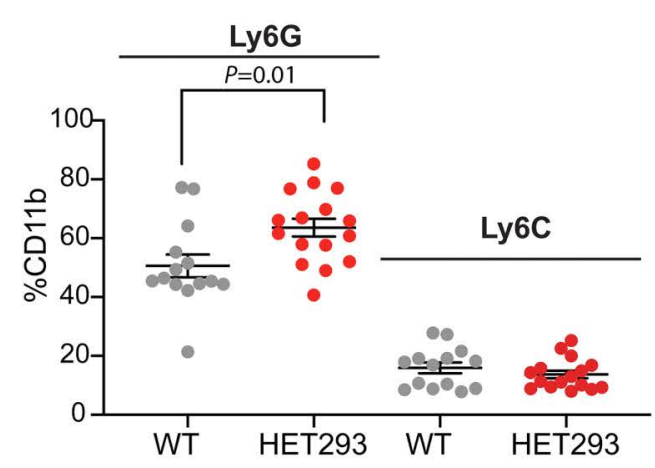

H

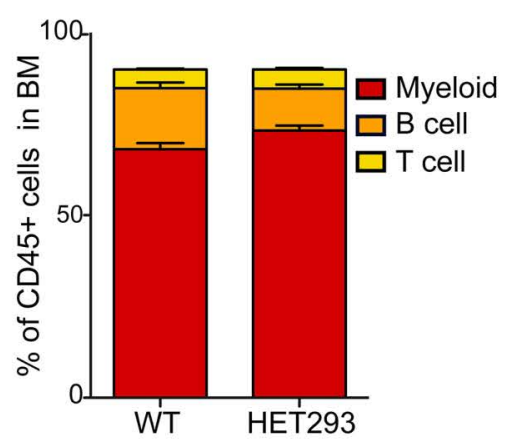

I

$\mathrm{J}$

K

L
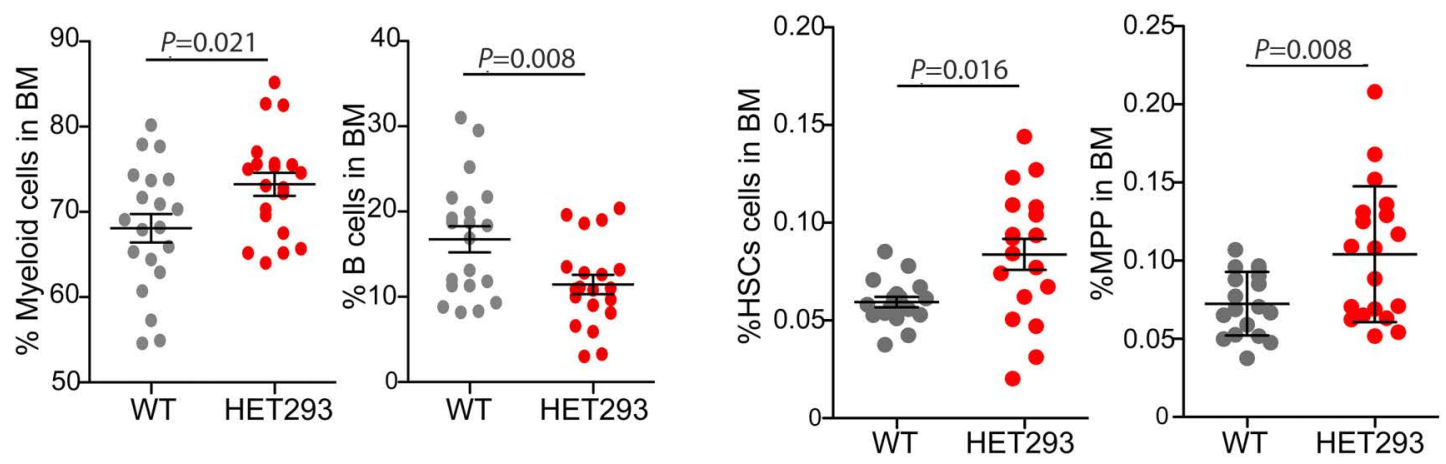

Figure 4. Tatton-Brown-Rahman syndrome mouse model characterized by myeloid expansion and increased frequency of hematopoietic stem and progenitor cells in the marrow. Complete blood cell counts performed on the blood of a representative cohort of mice with heterozygous in frame deletion of amino acid 293 of DNMT3A (HET293) $(n=23)$ and wild-type (WT) littermate controls $(n=31)$. All mice included in the analyses did not display hematologic malignancies. Displayed comparisons of $(A)$ total white blood cell (WBC) count, (B) percentage of neutrophils and (C) percentage of lymphocytes. Flow cytometric analysis of peripheral blood CD45 ${ }^{+}$leukocytes depicting (D) relative distribution of myeloid (defined as cells expressing CD11b and/or Ly6G), T cells (defined as CD3 and CD4 ${ }^{+}$and/or CD8 ${ }^{+}$cells) and B cells (defined as B220 cells) in the HET293 mice compared to WT. Quantification of the percentage of (E) myeloid and (F) B cells in the HET293 mice and WT mice as determined by flow cytometry from (D). (G) Analysis of the different subtypes of CD11 b myeloid cells into neutrophils (Ly6G expressing cells) or monocytes (Ly6C expressing cells). Flow cytometric analysis of bone marrow CD45 ${ }^{+}$leukocytes depicting H) relative distribution of myeloid, T cells and B cells in the HET293 mice compared to WT. Quantification of the percentage of I) myeloid and J) B cells in the HET293 mice and WT mice as determined by flow cytometry. Bone marrow flow cytometry assessment of HET293 and WT mice showing the percent of (K) hematopoietic stem/progenitor cells defined by SLAM markers and (L) multipotent progenitor (MPP) cells. 
germline deletion of Dnmt3a. ${ }^{27}$ The affected mice were moribund, with weight loss, pallor, adenopathy, tumors and splenomegaly (Figure 6A). Two mice had malignant myeloid neoplasms: one acute myeloid leukemia and one myeloproliferative neoplasm with infiltration of malignant cell into blood, bone marrow, liver and spleen. Four mice had lymphomas including B- $(n=3)$ and T-cell $(n=1)$ malignancies (Figure 6B). We also identified histolytic sarcoma in two mice, both of which had an additional malignancy (one with lymphoma and one with angiosarcoma). Two mice had angiosarcomas, a non-hematological malignancy (Online Supplementaty Figure S7).
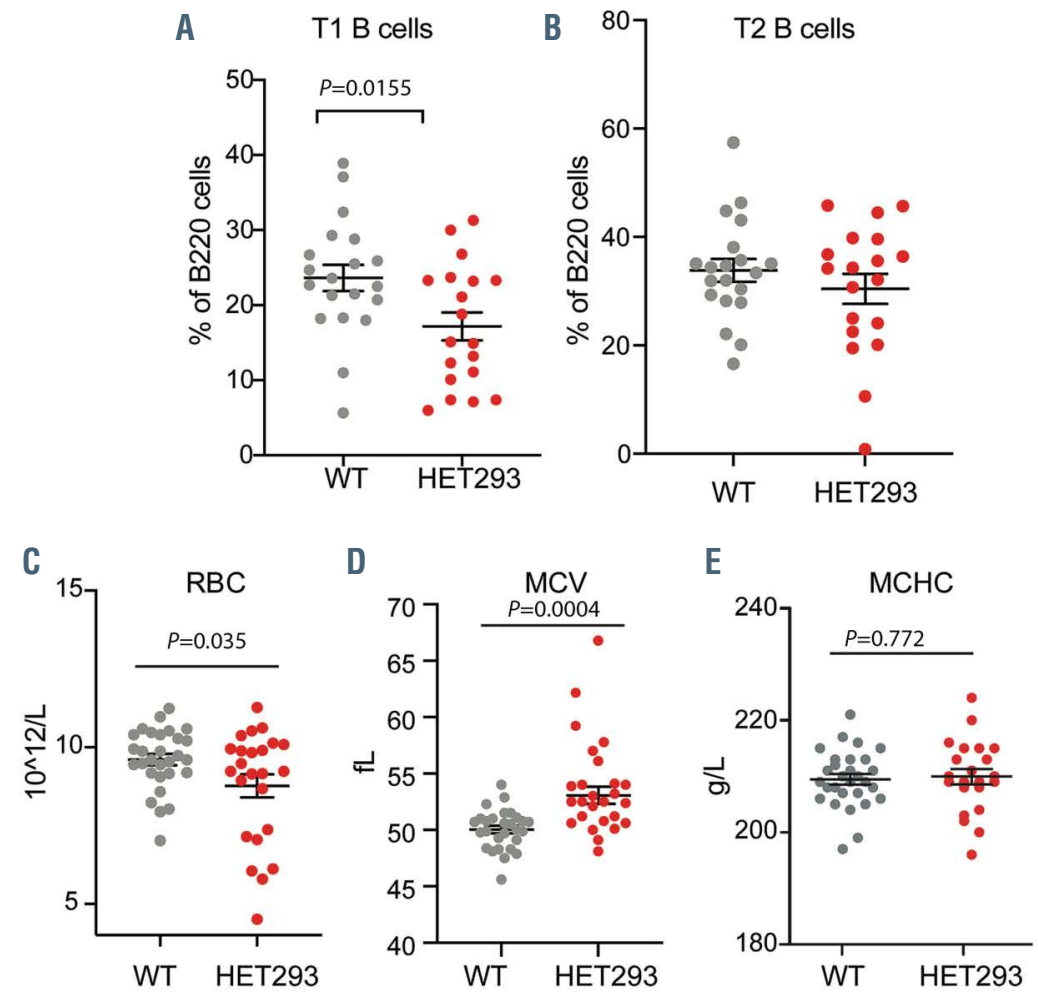

$\mathrm{F}$
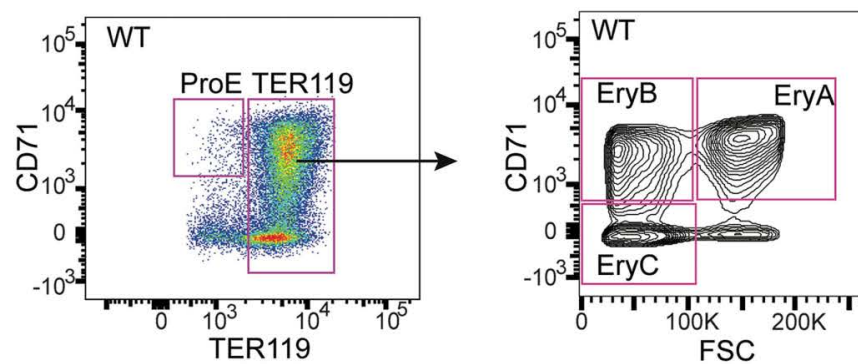

EryA - 32.6\%
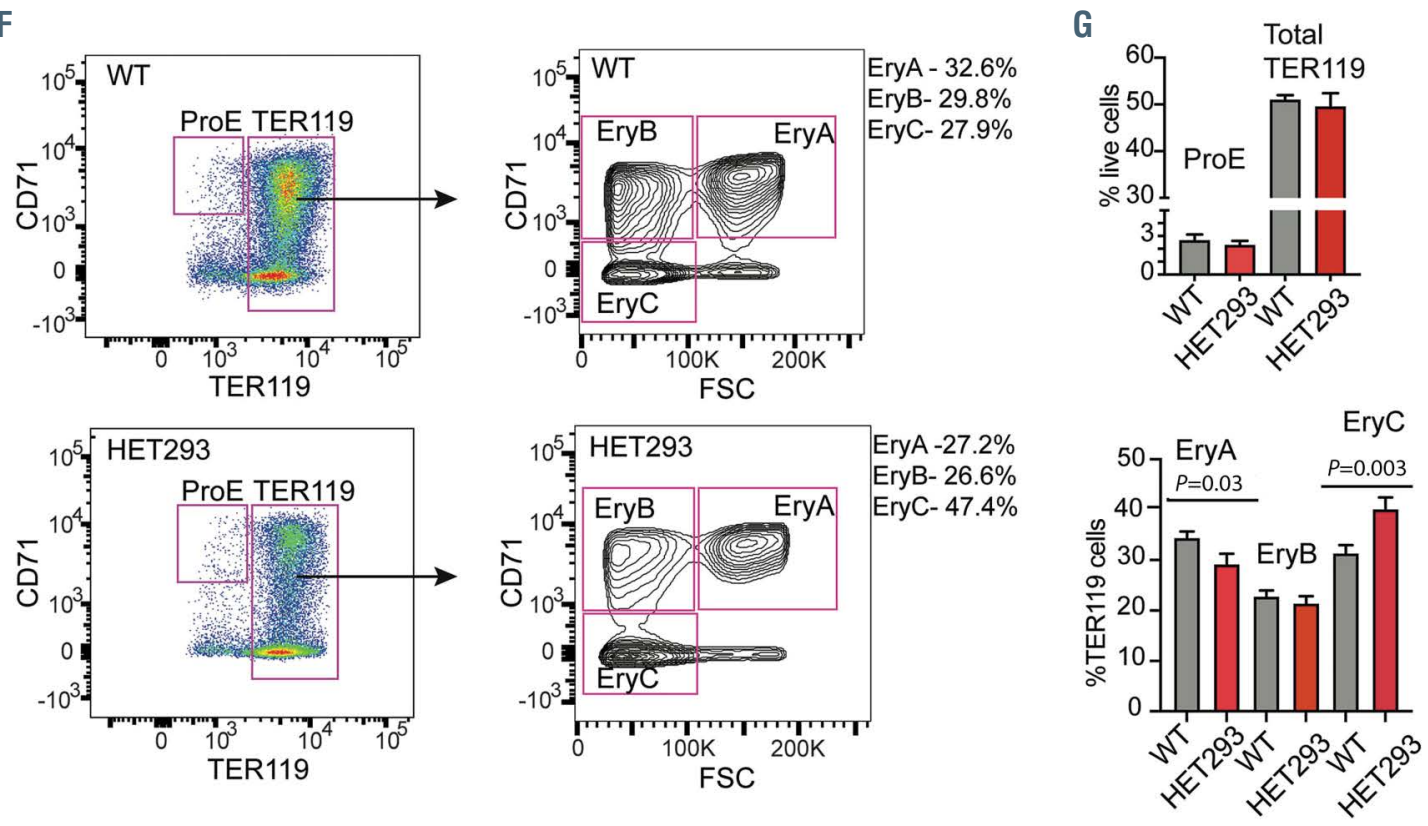

Figure 5. Differences in lymphoid and erythroid compartments in Tatton-Brown-Rahman syndrome mouse model. Quantification of the proportion of B220 ${ }^{+}$splenic $B$ cells that are (A) T1 B cells expressing immunglobulin (Ig) M and intermediate levels of IgD and (B) T2 B cells expressing both IgM and IgD in HET293 mice ( $=19$ ) and wild-type (WT) littermates $(n=20)$. From complete blood cell counts performed on peripheral blood, comparison of (C) red blood cell (RBC) number, (D) mean corpuscular volume (MCV), and (E) mean corpuscular hemoglobin concentration (MCHC) of WT mice $(n=29)$ and HET293 mice $(n=24)$. (F) Flow cytometric gating strategy for assessment of the erythroid development for representative WT and HET293 mice. Viable cells were gated based on their TER119 expression and then TER119+ ${ }^{+}$cells were plotted by forward scatter and CD71 expression levels to identify erythroblasts in different developmental stages (Ery A-C). (G) Top: the total proportion of TER119 and proerythroblasts (ProE) cells. Bottom: the proportion of the indicated populations within the TER119 ${ }^{+}$fraction defined by CD71 and FSC $(n=18)$ and HET293 mice $(n=16)$. 
These data, combined with case reports of individuals with TBRS and hematologic cancers, suggest that while the presence of a germline variant of DNMT3A increases the relative risk for the development of hematologic malignancies, a majority will not develop a blood cancer.

\section{Constitutive loss of DNMT3A in Tatton-Brown-Rahman syndrome leads to significant hypomethylation in hematopoietic cells}

In TBRS individuals only one functional allele of DNMT3A remains. In order to examine if constitutive loss of DNMT3A in TBRS impacts the DNA methylation landscape, we performed whole genome bisulfite sequencing on LCL derived from $B$ cells of a TBRS individual with a DNMT3A-297deletion (297del) and from a sibling of a
TBRS individual with WT DNMT3A. We measured a $6 \%$ decrease in global DNA methylation in the 297del LCL compared to WT LCL $(60.34 \%$ and $66.77 \%$, respectively) (Figure 7A). When we analyzed the distribution of DNA methylation in the WT LCL compared to the 297del LCL, we observed a significant decrease in DNA methylation in enhancer regions. This observation suggests that, similar to leukemia, ${ }^{28}$ in TBRS hematopoietic cells DNMT3A loss impacts methylation at regulatory enhancer regions (Figure 7B). We identified 1,068 differentially methylated regions (DMR) in 297del compared to WT LCL (Online Supplementary Table S8). Interestingly, some of these DMR include key loci with known importance in blood development such as the HOXA cluster, similar to the hypomethylation patterns we previously reported for

A

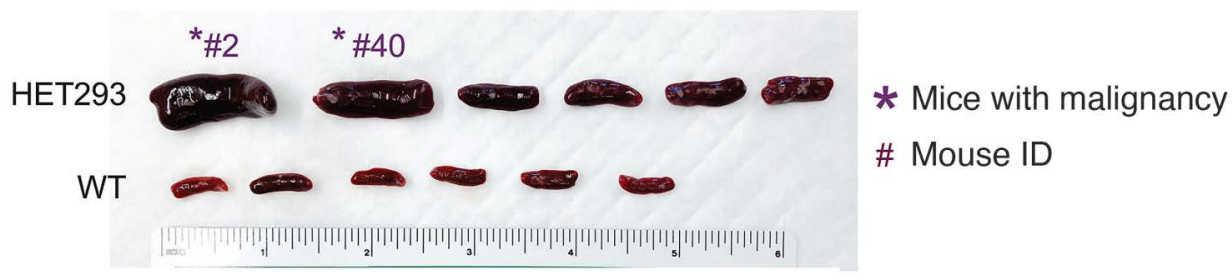

B

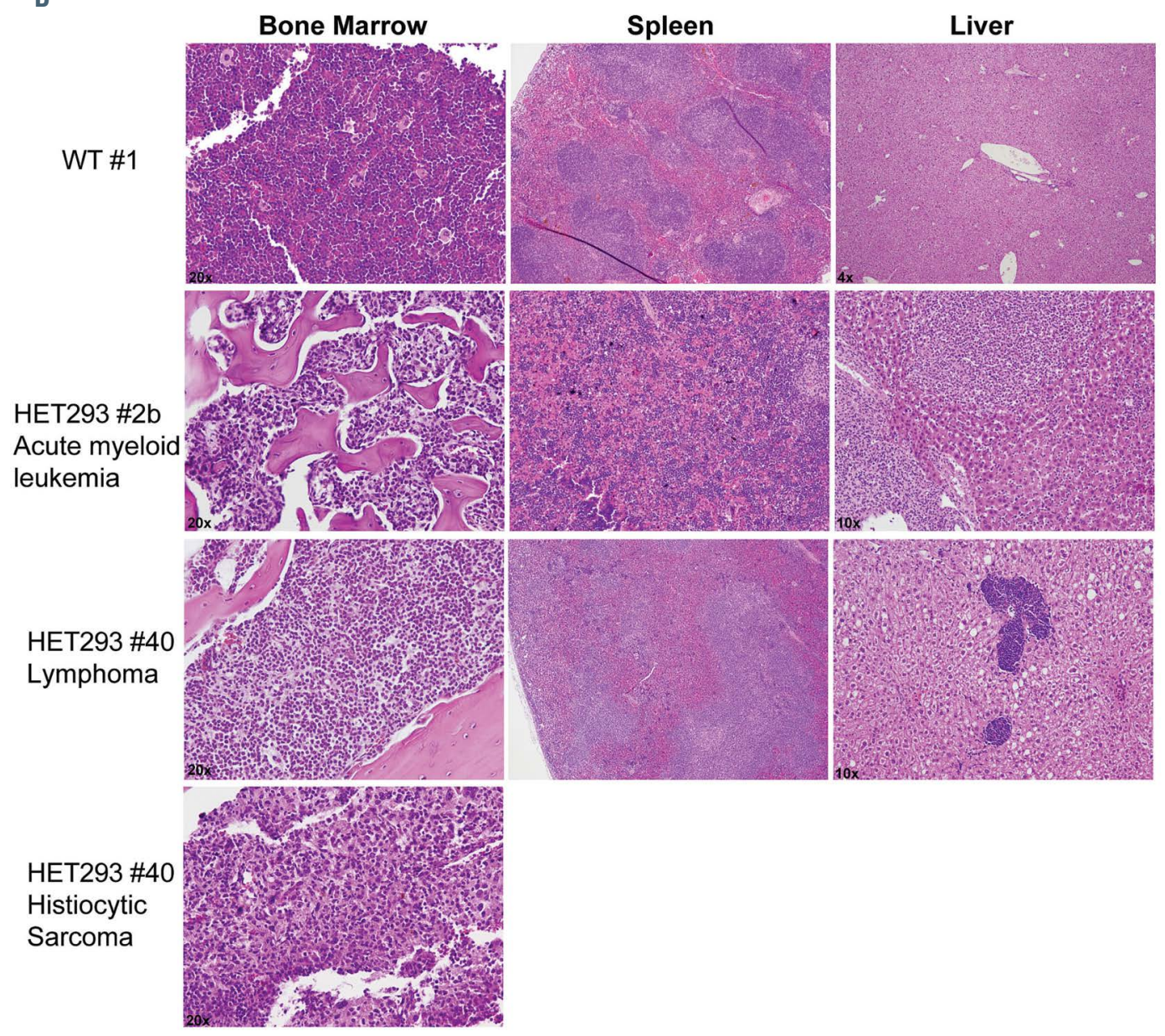

Figure 6. Development of hematologic malignancies in Tatton-Brown-Rahman syndrome mouse model. A subset of the HET293 mice had hematologic malignancies at 15 months of age. These mice were noted to have (A) enlarged spleens relative to wild-type (WT) and HET293 mice without malignancies. (B) Pathologic evaluation of the bone marrow, spleen and liver of a WT mouse (WT \#1, top row), a HET293 mouse with acute myeloid leukemia noted in the bone marrow, spleen and liver (HET293 2b, second row), and a HET293 with T-cell lymphoma in the bone marrow, spleen and liver (HET293 \#40, third row) and focal histiocytic sarcoma in the bone marrow (HET293 \#40, bottom image). 
A

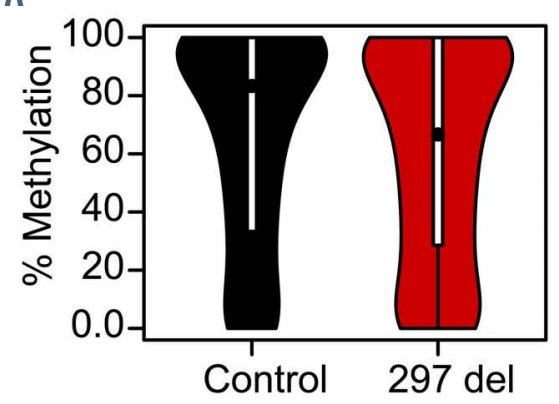

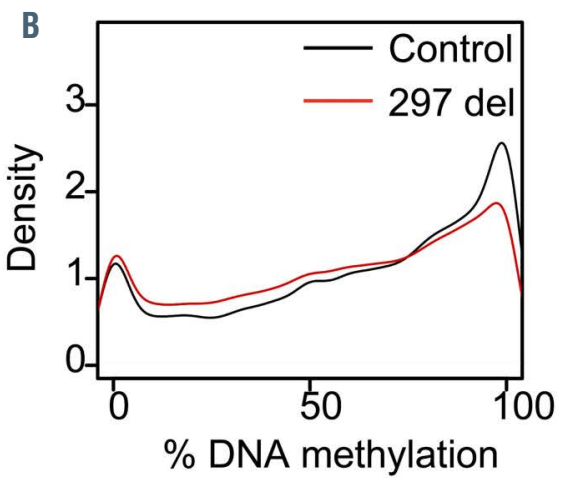

C

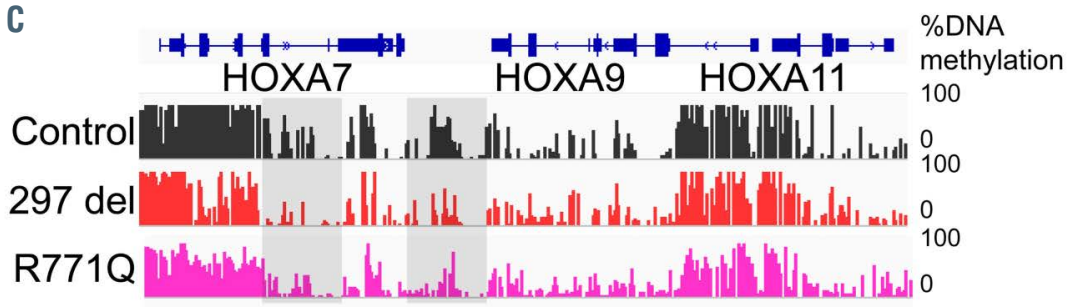

Figure 7. Altered DNA methylation in enhancer regions of hematopoietic cells of a Tatton-Brown-Rahman syndrome individual. Whole genome bisulfite sequencing was performed on a lymphoblastoid cell line (LCL) derived from a Tatton-Brown-Rahman syndrome (TBRS) individual with a heterozygous deletion of amino acid 297 in the PWWP domain of DNMT3A (297 del) and an LCL derived from the unaffected sibling of an individual with TBRS (control). (A) Overall \% CpG methylation of control and 297 de LCL. (B) Plot represents density of DNA CpG methylation of enhancer regions in control and 297 del LCL. (C) Genome browser tracks of $\mathrm{CpG}$ methylation at the HOXA locus of the control and 297 del LCL compared to a previously published DNMT3A p.R7710 mutant LCL.
LCLs generated from an individual with a constitutive DNMT3A 7710 mutation ${ }^{17}$ (Figure $7 C$ ). These results support that the heterozygous loss of DNMT3A leads to hematopoietic defects likely through altered DNA methylation.

\section{Discussion}

In order to examine the impact of constitutive loss of one copy of DNMT3A on blood production, we analyzed primary human specimens from patients with TBRS and murine models recapitulating pathogenic Dnmt $3 a$ variants identified in TBRS, CH and hematologic malignancies. Our data reveal a shift in the distribution of leukocytes with an overall increase in the myeloid compartment, specifically neutrophils, in individuals with TBRS compared to controls. Further, we identified a significant reduction in the percentage of lymphocytes and specific B- and T-cell subsets. We also noted erythropoiesis defects in TBRS, manifested as increased MCV. We further found that our TBRS murine models developed hematologic malignancies of low penetrance after a long latency. We also identified differences in the blood parameters of mice with differing Dnmt3a lesions (PWWP domain, ADD domain, or complete deletion of one allele), raising the possibility that mutation-specific effects on DNMT3A function may modify blood phenotypes. This work demonstrates that constitutive heterozygous loss of one DNMT3A allele leads to significant multilineage perturbations of hematopoiesis.

Our findings have potential clinical implications for individuals with TBRS. In our TBRS cohort and all examined TBRS mouse models, we found significantly reduced $B$ cells relative to controls. Additionally, one of our enrolled patients reported hypogammaglobulinemia requiring IVIG supplementation. Therefore, our observations suggest that evaluation of immunoglobulin levels may be warranted for individuals with TBRS, particularly those with recurrent infections. We also identified significant differences in T-cell subsets between TBRS indi- viduals and controls. In our TBRS cohort, the CD4/CD8 ratio was increased, a finding that has been associated with obesity which is noted in most of our TBRS cohort. ${ }^{29}$ Conversely, in our murine models, we found a decreased CD4/CD8 ratio in mice with Dnmt3a mutation or deletion. There are known differences in lymphoid development between human and mouse that may explain this discrepancy, but further investigations will be needed to fully understand the impact of DNMT3A loss on T-cell development. ${ }^{30}$ Lower CD4/CD8 ratios have been associated with altered immune responses and inflammation; perhaps this relative change might explain the increased level of inflammatory cytokine IL6 in our aged mutant mice. ${ }^{31}$ Overall, the clinical consequences of these immune cell phenotypes will require additional evaluation and longitudinal follow-up of affected individuals with a focus on how these B- and $T$ cell deficits impact susceptibility to infections and response to vaccinations.

In our cohort, we found increased MCV in TBRS individuals relative to controls. This finding is similar to the nonanemic macrocytosis characteristic of Down syndrome (DS) and Williams-Beuren syndrome (WBS). ${ }^{32,33}$ It is likely that in TBRS, like in DS and WBS, isolated macrocytosis is a benign variant of normal, warranting conservative observation only. Indeed, multiple individuals in our cohort had MCV consistently at or above the $90^{\text {th }}$ percentile for sex/age over many years yet have not developed any hematologic disorders. It is notable that increased red cell distribution width (RDW), reflecting variability in $\mathrm{RBC}$ size, is a consistent finding in $\mathrm{CH} .{ }^{9,21}$ Our data suggest that DNMT3A-mutant HSPC generate larger RBC than RBC derived from non-mutant HSPC. Therefore, in DNMT3Amutant $\mathrm{CH}$, increased $\mathrm{RDW}$ may be attributable to a mixed population of WT and DNMT3A-mutant cells in the peripheral blood. An expansion of the DNMT3A-mutant HSPC would therefore be expected to result in an increased MCV due to increased representation of $D N M T 3 A$-mutant $\mathrm{RBC}$ in the blood. Indeed, increased $\mathrm{MCV}$ in individuals with $\mathrm{CH}$ has been associated with increased risk of hematologic malignancy development. ${ }^{34}$ 
Unlike the TBRS patients, the aged HET293 mice were noted to have a modest, but significant decrease in RBC count compared to littermate controls. This may reflect a feature associated with aging and warrants further longitudinal studies on TBRS individuals to definitively determine changes in erythropoiesis over time.

In our TBRS cohort and murine models, we also identified neutrophil expansion, potentially in response to inflammation. $\mathrm{CH}$ is associated with increased risk not only for hematological malignancies, but also for cardiovascular disease, largely due to a pathologic inflammatory state. ${ }^{35}$ Previous studies have shown that loss in hematopoietic cells of Dnmt3a or Tet2, another commonly mutated gene in $\mathrm{CH}$, leads to increased expression of inflammatory chemokines and cytokines and accelerated atherosclerosis. ${ }^{21,36,37}$ Our cohort of TBRS individuals relative to unaffected controls did not show significantly increased IL6. However, it should be noted that our TBRS cohort included only children and young adults and it is possible an increase in inflammatory cytokines detectable by the relatively insensitive enzyme-linked immunosorbant assay may only be apparent with aging. If confirmed, increased inflammation with aging in TBRS individuals may elevate the risk of atherosclerotic cardiovascular disease, warranting lifetime monitoring. This monitoring may be of particular importance given that TBRS is associated with congenital cardiac defects. ${ }^{1,37}$

Given the association between somatic DNMT3A mutations and leukemia, malignancy risk is a major concern for TBRS individuals. While there are case reports in the literature of TBRS individuals developing hematologic malignancies, the absolute risk is currently undefined. In this study we found that unperturbed HET293 mice developed spontaneous hematologic malignancies at higher rates than WT mice, consistent with constitutive Dnmt3a-HET and previous studies using complete somatic Dnmt3a deletion. ${ }^{27,38,39}$ We also identified malignancies which are not often associated with DNMT3A-mutations and not reported to date in TBRS; histolytic sarcoma and angiosarcoma. Interestingly, pathologic examination of non-moribund aged HET293 mice revealed extramedullary hematopoiesis (EMH) involving spleen, liver and kidney in a subset of mice (Online Supplementary Figure S7). EMH can be a feature of myeloproliferative neoplasm. ${ }^{40}$ However, in our TBRS models this finding is of uncertain significance as the affected HET293 mice were healthy without significant blood count abnormalities. Overall, our findings support a possible increased risk for hematologic malignancies in individuals with TBRS. Thus, TBRS individuals and their families should be educated about the signs and symptoms of leukemia that would warrant medical evaluation. However, the relatively low penetrance of malignancy in our mouse model, even at advanced ages, indicates that leukemia is not an inevitable consequence of having a constitutive DNMT3A mutation. Indeed, our previous report demonstrated that, although loss of DNMT3A leads to a significant competitive advantage in the blood, this advantage is not necessarily associated with leukemogenesis. ${ }^{17}$ Natural history studies of TBRS individuals are needed to define the incidence of hematologic malignancy, the disease spectrum, and risk predictors for malignancy development to enable tailored surveillance guidelines for prospective monitoring.

In conclusion, we show that constitutive loss of DNMT3A significantly impacts multilineage blood development and leads to phenotypic changes with clinical implications. While our findings offer important insights into blood development in individuals with TBRS, these results may also have broader implications. The DNMT3A mutations in TBRS and CH largely overlap and, therefore, TBRS may offer a unique opportunity to address the impact of $D N M T 3 A$ mutations on hematopoiesis in a state mimicking accelerated $\mathrm{CH}$.

\section{Disclosures \\ No conflicts of interest to disclose.}

\section{Contributions}

AT, MAG and RER conceived the project, discussed and designed experiments; $A T$ performed experiments and analysis with assistance of $C R, G M, L Z, K K, S E C, A G G, R A$ and $C W C ; A G S, A G$ and $A N M$ performed flow cytometry analysis on TBRS individuals; ANM and MJH performed all the pathological analysis in this manuscript; YHH, TA and AT generated the mouse models for all studies; JJK, MTC, ALA, IV, RS, LVM and $L B$ assisted with collection of blood and medical data from TBRS patients; JMR conducted the bioinformatic analyses in the manuscript; AT, MAG and RER wrote the manuscript; all authors interpreted the results and edited the manuscript.

\section{Acknowledgments}

We thank our research participants and their families for their contributions and active involvement. We thank the TBRS Community for their kind help in supporting this project. We especially wish to thank the TBRS Community board of directors, particularly Jill Kiernan and Kerry Grens. We also thank C. Gillespie for critical review.

\section{Funding}

This project was supported by the Baylor College of Medicine's Human Stem Cell Core and Baylor College of Medicine's Cytometry and Cell Sorting Core, which are funded in part by the institution and the NIH CA125123, OD028591, AI036211, A125123, RR024574, DK092833, CA183252, K08CA201611 and the HHMI James H. Gilliam Fellowships. Publication costs were generously supported by the Texas Children's Hospital Young Investigators Endowed Fund.

\section{References}

1. Tatton-Brown K, Zachariou A, Loveday C, et al. The Tatton-Brown-Rahman syndrome: a clinical study of 55 individuals with de novo constitutive DNMT3A variants. Wellcome Open Res. 2018;3:46.

2. Tatton-Brown K, Seal S, Ruark E, et al. Mutations in the DNA methyltransferase gene DNMT3A cause an overgrowth syn- drome with intellectual disability. Nat Genet. 2014;46(4):385-388

3. Challen GA, Sun D, Jeong M, et al. Dnmt3a is essential for hematopoietic stem cell differentiation. Nat Genet. 2011;44(1):23-31.

4. Yang L, Rau R, Goodell MA. DNMT3A in haematological malignancies. Nat Rev Cancer. 2015;15(3):152-165.

5. Ley TJ, Ding L, Walter MJ, et al. DNMT3A mutations in acute myeloid leukemia. N Engl J Med. 2010;363(25):2424-2433.
6. Brunetti L, Gundry MC, Goodell MA DNMT3A in leukemia. Cold Spring Harb Perspect Med. 2017;7(2):a030320.

7. Genovese G, Kahler AK, Handsaker RE, et al. Clonal hematopoiesis and blood-cancer risk inferred from blood DNA sequence. $\mathrm{N}$ Engl J Med. 2014;371(26):2477-2487.

8. Jaiswal S, Fontanillas P, Flannick J, et al Age-related clonal hematopoiesis associated with adverse outcomes. $\mathrm{N}$ Engl J Med. 2014;371(26):2488-2498. 
9. Abelson S, Collord G, Ng SWK, et al. Prediction of acute myeloid leukaemia risk in healthy individuals. Nature. 2018; 559(7714):400-404

10. Bolouri H, Farrar JE, Triche T, Jr., et al. The molecular landscape of pediatric acute myeloid leukemia reveals recurrent structural alterations and age-specific mutational interactions. Nat Med. 2018;24(1):103112.

11. Ho PA, Kutny MA, Alonzo TA, et al. Leukemic mutations in the methylationassociated genes DNMT3A and IDH2 are rare events in pediatric AML: a report from the Children's Oncology Group. Pediatr Blood Cancer. 2011:57(2):204-209.

12. Liu Y, Easton J, Shao Y, et al. The genomic landscape of pediatric and young adult T-lineage acute lymphoblastic leukemia. Nat Genet. 2017;49(8):1211-1218.

13. Zaliova M, Stuchly J, Winkowska L, et al. Genomic landscape of pediatric B-other acute lymphoblastic leukemia in a consecutive European cohort. Haematologica. 2019:104(7):1396-1406.

14. Hollink I, van den Ouweland AMW, Beverloo HB, Arentsen-Peters S, Zwaan CM, Wagner A. Acute myeloid leukaemia in a case with Tatton-Brown-Rahman syndrome: the peculiar DNMT3A R882 mutation. J Med Genet. 2017;54(12):805-808.

15. Community T. 2020 [cited; Available from: https://tbrsyndrome.org/

16. Huang Y, Tovy A, Sundaramurthy V, et al. Nearly a third of clonal hematopoiesisassociated DNMT3A mutations reduce protein stability and may be associated with poorer prognosis. Blood. 2018; 132(Suppl 1):S1315.

17. Tovy A, Reyes IM, Gundry MC, et al. Tissue-biased expansion of DNMT3Amutant clones in a mosaic individual is associated with conserved epigenetic erosion. Cell Stem Cell. 2020;27(2):326-335.

18. Chen $H$, Smith $A D$, Chen T. WALT: fast and accurate read mapping for bisulfite sequencing. Bioinformatics. 2016; 32(22):3507-3509.

19. Ladle BH, Li KP, Phillips MJ, et al. De novo DNA methylation by DNA methyltrans- ferase 3a controls early effector CD8+ Tcell fate decisions following activation. Proc Natl Acad Sci U S A. 2016;113(38):10631 10636.

20. Watson CJ, Papula AL, Poon GYP, et al. The evolutionary dynamics and fitness landscape of clonal hematopoiesis. Science. 2020;367(6485):1449-1454.

21. Bick AG, Weinstock JS, Nandakumar SK, et al. Inherited causes of clonal haematopoiesis in 97,691 whole genomes. Nature. 2020;586(7831):763-768.

22. Jeong M, Park HJ, Celik $\mathrm{H}$, et al. Loss of Dnmt3a immortalizes hematopoietic stem cells in vivo. Cell Rep. 2018;23(1):1-10.

23. Petro JB, Gerstein RM, Lowe J, Carter RS, Shinners N, Khan WN. Transitional type 1 and 2 B lymphocyte subsets are differentially responsive to antigen receptor signaling. J Biol Chem. 2002;277(50):4800948019.

24. Loder F, Mutschler B, Ray RJ, et al. B cell development in the spleen takes place in discrete steps and is determined by the quality of B cell receptor-derived signals. J Exp Med. 1999;190(1):75-89.

25. Socolovsky M, Nam H, Fleming MD Haase VH, Brugnara C, Lodish HF Ineffective erythropoiesis in Stat5a(-//)5b $(-/-)$ mice due to decreased survival of early erythroblasts. Blood. 2001;98(12):32613273

26. Koulnis M, Pop R, Porpiglia E, Shearstone IR, Hidalgo D, Socolovsky M. Identification and analysis of mouse erythroid progenitors using the CD71/TER119 flow-cytometric assay. I Vis Exp. 2011;(54):2809

27. Cole CB, Russler-Germain DA, Ketkar S, et al. Haploinsufficiency for DNA methyltransferase $3 \mathrm{~A}$ predisposes hematopoietic cells to myeloid malignancies. J Clin Invest. 2017;127(10):3657-3674

28. Spencer DH, Russler-Germain DA, Ketkar $\mathrm{S}$, et al. $\mathrm{CpG}$ island hypermethylation mediated by DNMT3A is a consequence of AML progression. Cell. 2017;168(5):801816.

29. van der Weerd K, Dik WA, Schrijver B, et al. Morbidly obese human subjects have increased peripheral blood CD4+ T cells with skewing toward a Treg- and Th2 dominated phenotype. Diabetes. 2012;61(2):401-408.

30. Kumar BV, Connors TJ, Farber DL. Human T cell development, localization, and function throughout life. Immunity. 2018; 48(2):202-213.

31. McBride JA, Striker R. Imbalance in the game of $\mathrm{T}$ cells: what can the CD4/CD8 Tcell ratio tell us about HIV and health? PLoS Pathog. 2017;13(11):e1006624.

32. Wachtel TJ, Pueschel SM. Macrocytosis in Down syndrome. Am J Ment Retard. 1991;95(4):417-420.

33. Yu E, Feinn R, Bona R, et al. Mild macrocytosis in Williams-Beuren syndrome. Eur Med Genet. 2020;63(3):103740.

34. Sperling AS, Gibson CJ, Ebert BL. The genetics of myelodysplastic syndrome: from clonal haematopoiesis to secondary leukaemia. Nat Rev Cancer. 2017;17(1):519.

35. Steensma DP, Ebert BL. Clonal hematopoiesis as a model for premalignant changes during aging. Exp Hematol. 2020 83:48-56.

36. Fuster JI, MacLauchlan S, Zuriaga MA, et al. Clonal hematopoiesis associated with TET2 deficiency accelerates atherosclerosis development in mice. Science. 2017;355 (6327):842-847.

37. Jaiswal S, Natarajan P, Ebert BL. Clonal hematopoiesis and atherosclerosis. $\mathrm{N}$ Engl J Med. 2017:377(14):1401-1402.

38. Celik H, Mallaney C, Kothari A, et al Enforced differentiation of Dnmt3a-nul bone marrow leads to failure with c-Kit mutations driving leukemic transformation. Blood. 2015;125(4):619-628.

39. Mayle A, Yang L, Rodriguez B, et al Dnmt3a loss predisposes murine hematopoietic stem cells to malignant transformation. Blood. 2015;125(4):629638.

40. Fan N, Lavu S, Hanson CA, Tefferi A Extramedullary hematopoiesis in the absence of myeloproliferative neoplasm: Mayo Clinic case series of 309 patients. Blood Cancer J. 2018;8(12):119. 